\title{
Coagulation studies in rheumatic heart disease
}

\author{
J L TOY,* D A Lederer, A T TUlpule, A P TANDON, S H TAYLOR, \\ G P MCNICOL
}

From the University Departments of Medicine and Cardiovascular Studies, The General Infirmary, Leeds

SUMMARY The clotting characteristics of pulmonary and systemic blood were studied in 10 patients with chronic rheumatic mitral valve disease complicated by atrial fibrillation and in seven patients with aortic valve disease in sinus rhythm. A haemostatic basis for the association of rheumatic mitral valve disease with thrombotic emboli was sought.

Both groups of patients showed differences in platelet function between pulmonary and systemic arterial blood. In patients with mitral valve disease aggregation of platelets was significantly greater in pulmonary than in systemic arterial blood at rest; the converse was true during exercise. In aortic valve disease platelet aggregation was greater in systemic than in pulmonary arterial blood at all times. Only the patients with mitral valve disease showed changes in blood coagulation during passage through the lungs and left heart; there was a small but statistically significant shortening in partial thromboplastin time in systemic as compared with pulmonary arterial blood both at rest and during exercise. Similarly, the effects of exercise on the various haemostatic factors measured were largely confined to the patients with mitral valve disease; in these patients exercise stimulated an increase in factor VIII in pulmonary arterial blood and an increase in platelet adhesiveness and aggregability in left heart blood. These changes provide a basis for the suggestion that in patients with rheumatic mitral valve disease, unlike those with aortic valve disease, there is an increased thrombotic tendency in blood in the left heart which is particularly pronounced during exercise.

Systemic emboli are a serious hazard of rheumatic mitral valve disease. ${ }^{12}$ The risk does not appear to be related to the type of valvular deformity, ${ }^{3}$ or haemodynamic derangement. ${ }^{4}$ Though relevant, few studies have been reported of possible abnormalities in the clotting characteristics of blood as it passes across the damaged mitral valve. The following study was undertaken to determine whether such abnormalities exist.

\section{Subjects and methods}

Seventeen patients were studied. Ten, including six women, had moderate or severe mixed mitral valve disease complicated in all by chronic atrial fibrillation. Seven patients, including four women, with mild aortic valve disease in sinus rhythm furnished a control population. Clinical diagnosis in all was confirmed by angiographic and haemo-

$\star$ Present address : Imperial Cancer Research Fund, Lincoln's Inn Fields, London W2

Received for publication 17 July 1979 dynamic studies. No patient was in congestive heart failure, none had a history of embolic episodes, and none had taken anticoagulants. All gave their consent for the study which was agreed by the hospital ethical committee.

\section{DESIGN OF STUDY}

(a) In vitro studies

These were undertaken to determine the effect of the passage of blood through the catheters used in the in vivo study. Peripheral venous blood was taken from eight healthy volunteers (four men, four women) into plastic syringes without anticoagulation. Blood was taken into three syringes; from the first syringe blood was transferred direct to $1 / 9$ volume 3.8 per cent sodium citrate, and from the second and third syringes was passed, respectively, through the pulmonary $(8 \mathrm{~F}$ US Catheter Co. Ltd.) or the arterial $(75 \times 0.2 \mathrm{~cm}$ Portex Ltd.) catheter. The first $5 \mathrm{ml}$ blood passing through each catheter were discarded and the remainder collected in the appropriate tubes for platelet and coagulation factor measurements. 
(b) Comparison of blood clotting variables in pulmonary and systemic arterial circulations Measurements were made at the time of routine diagnostic haemodynamic evaluation. Blood samples were withdrawn simultaneously from the pulmonary and systemic arteries at rest and at the end of two minutes of submaximum supine bicycle exercise. Pulmonary arterial blood was withdrawn through a woven Dacron catheter (8F US Catheter Co. Ltd.) and aortic blood through a nylon catheter $(75 \times 0.2 \mathrm{~cm}$ Portex Ltd.). No anticoagulants were used; catheters were kept patent by saline infusion.

The first $10 \mathrm{ml}$ blood withdrawn from each catheter were discarded. Samples were then taken into plastic syringes and mixed with heparin (40 IU/ $10 \mathrm{ml}$ ), for platelet adhesion studies, with 1 per cent tranexamic acid for fibrin related antigen measurement, with EDTA for platelet counts, and with $1 / 9$ volume 3.8 per cent sodium citrate for the remaining tests.

The citrated blood was spun at $250 \mathrm{~g}$ for 15 minutes to obtain platelet rich plasma which was drawn off with a siliconised pipette. The remainder of the blood was spun at $2500 \mathrm{~g}$ for 20 minutes at $4^{\circ} \mathrm{C}$ to obtain platelet poor plasma.

Platelet counts were performed on a Coulter thrombocounter. Coagulation studies were performed by the following methods:

Activated partial thromboplastin time ${ }^{5}$; fibrinogen $^{6}$; plasminogen ${ }^{7 \mathrm{7a}}$; urokinase sensitivity ${ }^{8}$; euglobulin lysis time ${ }^{9}$; fibrinogen related antigen ${ }^{10}$; factor VIII ${ }^{11}$; platelet adhesiveness ${ }^{12}$; platelet aggregation $^{13}$; platelet factor $3 .^{14}$

Platelet aggregation was measured in response to adrenaline (acid bitartrate, Sigma Chemical Co., St. Louis), to ADP (Sigma Chemical Co., St. Louis), and to soluble collagen. ${ }^{15}$ These agents were diluted in tris-buffered saline $\mathrm{pH} \mathrm{7.4}$ and added to the platelet rich plasma in amounts to give final concentrations of $5 \mu \mathrm{mol}$ adrenaline and $0 \cdot 5,2 \cdot 0$, and $4.0 \mu \mathrm{mol}$ ADP. A quantitative assessment of platelet aggregation was made by measuring the slope of the changes in light transmission (IR), the amount of light transmission three minutes after addition of the aggregation agent $\left(\mathrm{T}_{3}\right)$, and the maximal light transmission achieved ( $\mathrm{T} \max$ ) within three minutes.

\section{Results}

\section{CATHETER EFFECTS ON BLOOD CLOTTING VARIABLES}

The results are summarised in Table 1. No statistically significant difference was noted in any of the indices studied between the blood that was
Table 1 Comparison of effects of passage of non-anticoagulated peripheral venous blood from healthy volunteers through pulmonary and arterial catheters (mean values \pm standard error of mean)

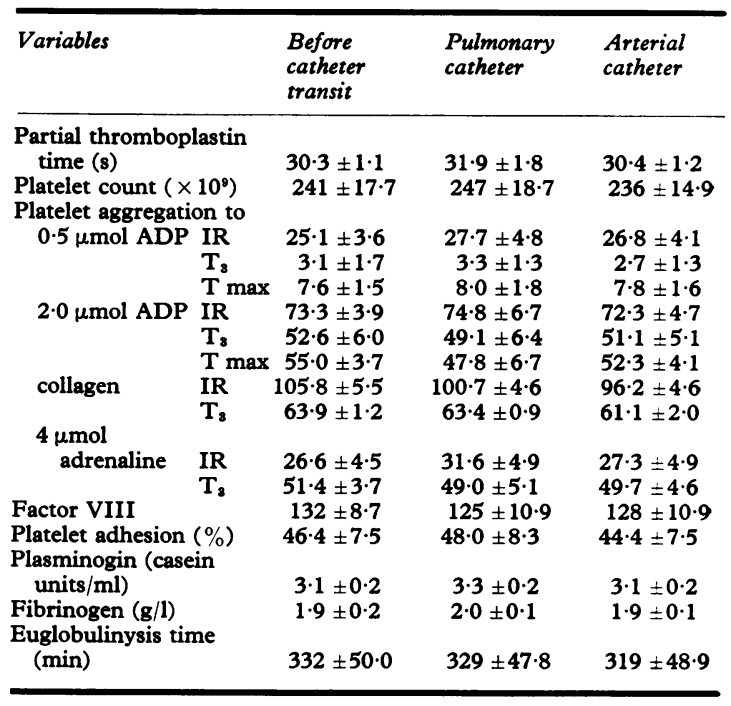

No statistically significant difference was noted between the three sets of determination.

and was not passed through arterial or pulmonary catheters.

COMPARISON OF HAEMOSTATIC FACTORS IN PULMONARY AND SYSTEMIC ARTERIAL BLOOD

Factors in which there was a statistically significant difference are detailed in Table 2.

In mitral valve disease

At rest: there was a small decrease in activated partial thromboplastin time and a decrease in platelet aggregation in aortic as compared with pulmonary arterial blood.

During exercise: the small atrioventricular difference in PTT was maintained accompanied by a significant decrease in platelets and an increase in platelet aggregation (to $2.0 \mu \mathrm{mol} A D P \mathrm{~T}$ max) in aortic as compared with pulmonary arterial blood.

In aortic valve disease

At rest: there was a significant increase in platelet aggregation in aortic as compared with pulmonary arterial blood.

During exercise : the increase in platelet aggregation in aortic blood was maintained.

CHANGES IN HAEMOSTATIC FACTORS

INDUCED BY EXERCISE

Factors in which there was a statistically significant 
Table 2 Statistically significant changes in haemostatic measurements : effects of transit through pulmonary circulation and left heart-mean values are presented

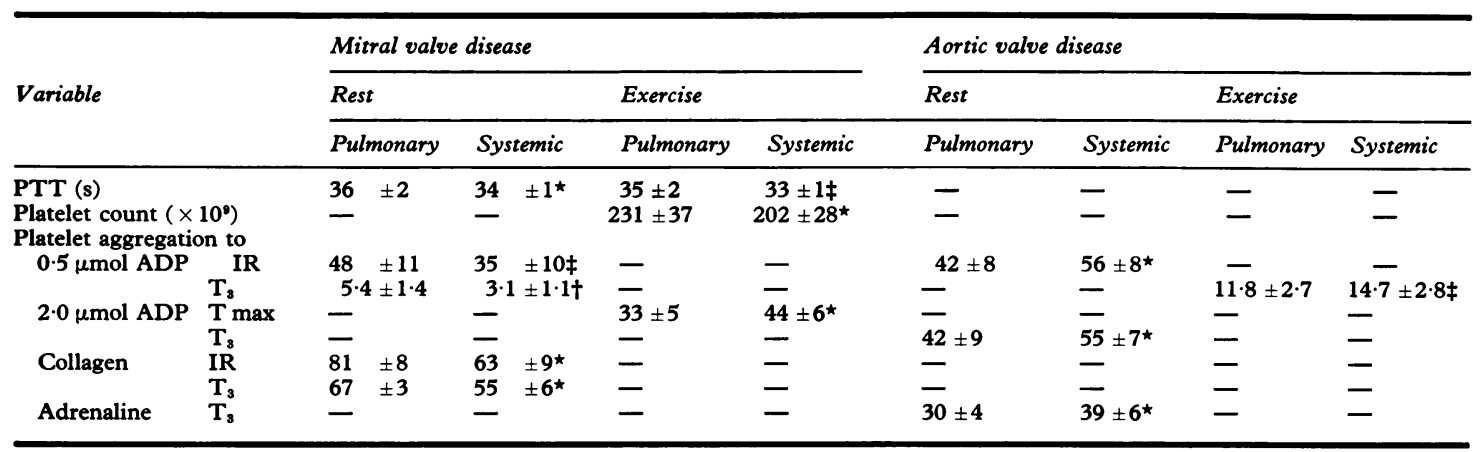

${ }^{\star} \mathrm{p}<0.05 ; \dagger<0.02 ; \ddagger<0.01$.

difference are detailed in Table 3.

\section{In mitral valve disease}

Exercise was associated with an increase in factor VIII in pulmonary arterial blood and an increase in platelet adhesion and aggregation in systemic arterial blood.

In aortic valve disease

Exercise was associated with an increase in platelet aggregation in pulmonary arterial blood; there were no changes in any of the other haemostatic factors measured in either pulmonary or aortic blood.

\section{Discussion}

These observations demonstrate a number of differences between the clotting factors in the pulmonary and systemic arterial blood of patients with advanced mitral valve disease and those in a control population of patients with mild aortic valve disease. As shown in Table 1, passage through the different catheters produced only trivial and statistically insignificant changes in the haemostatic function tests.
The most pronounced changes found in the patient studied were seen in platelet function. At rest platelet aggregation was less in systemic than in pulmonary arterial blood in patients with mitral valve disease; in patients with aortic valve disease the converse was true. During exercise both groups showed an increase in platelet aggregation in systemic as compared with pulmonary arterial blood, and in the patients with mitral valve disease this was accompanied by a diminution in aortic platelet count. Exercise was responsible for a pronounced increase in platelet adhesion and aggregation in aortic blood in mitral valve disease; in contrast, changes in aortic valve disease were confined to a limited increase in aggregation in pulmonary blood.

Before attempting to interpret these complex changes it is well to examine the limitations imposed by the design of the study. None of the patients with mitral valve disease had had emboli; the haemostatic factors in this cohort may, therefore, be quite different from those in a group of patients who had suffered such an event. The control group of patients with aortic valve disease was chosen for ethical reasons. However, their valve lesion was

Table 3 Statistically significant changes in haemostatic measurements: effects of exercise-mean values are presented

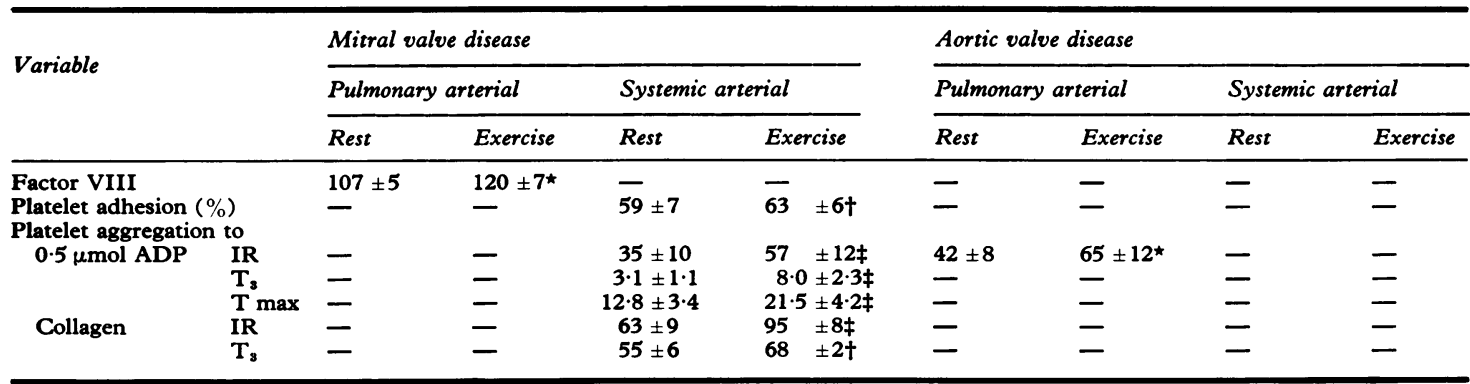

${ }^{\star} \mathrm{p}<0.5 ; \quad$ tp $<0.02 ; \quad \neq \mathrm{p}<0.01$. 
mild, all were in sinus rhythm, and their clinical state was such as to warrant their inclusion as reasonable control population. It could also be argued that a higher workload or longer periods of exercise would have induced more pronounced changes. This may indeed be true, but both were at the limits ethically justifiable during investigation of patients with mitral valve disease. Finally, it must be emphasised that the pulmonary systemic arterial differences in blood clotting factors measured describe only composite changes; they allow no separation of the particular effects of transit through the lesser circulation from those occurring during passage through the left atrium and over the damaged mitral valve.

Despite these design limitations, the results are interesting. The reduction in platelet aggregation after passage through the pulmonary circulation and left heart at rest in patients with mitral valve disease contrasts with the reverse situation in aortic valve disease. However, during exercise which can be expected to increase platelet stickiness, ${ }^{16}$ there was an increase in platelet aggregation in systemic as compared with pulmonary arterial blood in both groups; in the patients with mitral valve disease there was additionally a coincidental reduction in the systemic arterial platelet count. The interpretation of these changes in pathological terms is speculative. It can be reasoned that in the patients with mitral valve disease at rest, many of whom have shortened platelet survival, ${ }^{17}$ the more sticky platelets were sequestered in the pulmonary blood vessels or left heart during the retarded passage of blood through the lesser circulation. There is a substantial incidence of pulmonary arterial intimal damage and atheroma in patients with pulmonary hypertension caused by mitral valve disease; such intimal lesions could be expected to form a nidus for deposition of the most sticky platelets. There is no large platelet pool in the lesser circulation, ${ }^{18}$ so that the more rapid flow of blood through the lungs during exercise could be expected to reverse this trend, as was observed. However, these speculations are at variance with the observations of Kallinkos-Maniatis, ${ }^{19}$ who found a significantly greater platelet count in systemic as compared with central venous blood in patients with a range of cardiac diagnoses. The difference in our findings could possibly be explained by differences in the techniques of measurement used and the patients in the two studies.

Recently published observations ${ }^{20}{ }^{21}$ have shown that in a number of animal species the lungs are the site of continuous generation of prostacyclin $\left(\mathrm{PGI}_{2}\right)$ the most potent inhibitor of platelet aggregation so far described, and that vigorous hyperventilation increases release from the lungs of a platelet disaggregating agent. Piper and Vane ${ }^{22}$ showed that injection of particles into guinea-pig lungs led to release of prostaglandins, except when the injections led to an increase in pulmonary arterial pressure when there was no detectable release of prostaglandins. It may be that our studies in man can be explained on the basis that the aortic valve disease 'control' patients mimic the general animal response cited above and that mitral valve disease patients with their damaged lungs and pulmonary arterial hypertension at rest mimic the guinea-pigs and only behave as the aortic valve disease patients do after the stimulus of exercise and the associated increase in ventilation.

The only other difference in haemostatic changes between the two groups was in the blood coagulation screening test, PTT. Activated PTT was less in systemic than in pulmonary arterial blood both at rest and during exercise in patients with aortic valve disease. In the group with mitral valve disease exercise also increased factor VIII activity, confirming other reports. ${ }^{23}$ Again, though the pathological interpretation of these findings is speculative, the increase in factor VIII activity during exercise and the reduction in PTT during passage of blood through the lesser circulation could possibly be expected to increase the tendency to thrombus formation, particularly in atheromatous pulmonary vessels or left atrium, the seat of endocardial damage or excessive blood stasis.

These studies give no clue as to the mechanisms involved in these platelet and blood coagulability changes in patients with mitral valve disease, but they prompt an interesting speculation. The prime characteristic of impaired cardiac function is reflex activation of the sympathoadrenal system and a raised concentration of catecholamines in the plasma. ${ }^{24}$ These changes are particularly noticeable in patients with mitral valve disease and increase during exertion. Such sympathoadrenal activation can be expected to stimulate the breakdown of energy-rich ATP to ADP in the tissues and blood cells and thus enhance the adhesiveness of platelets and their tendency to aggregation.

Whatever the mechanisms involved, our findings are consistent with the fact that patients with mitral valve disease do indeed have an increased tendency to platelet deposition and thrombin formation followed by fibrin deposition in the lesser circulation and left heart. This is not to imply that such changes are the primary cause of clotting events in these patients. It is much more likely that the tardy blood flow, the high pressures in the pulmonary vessels and left atrium, and the intimal damage in these vascular compartments are the basic pathogenic 
factors. However, our results are fully compatible with the haemostatic changes that could be expected in such a situation. They also give theoretical support to the practical therapeutic observations that in mitral valve disease anticoagulants reduce the incidence of arterial embolism, ${ }^{125}$ and that antiplatelet agents do so in patients after mitral valve surgery. ${ }^{26}$ Clearly, more work is necessary, and results give impetus for such endeavours.

We are grateful to the Special Trustees of the General Infirmary for financial support.

\section{References}

'Fleming HA, Bailey SM. Mitral valve disease, systemic embolism and anticoagulants. Postgrad Med F 1971; 47: 599-604.

${ }^{2}$ Selzer A, Cohn KE. Natural history of mitral stenosis. A review. Circulation 1972; 45: 878-90.

${ }^{3}$ Coulshed D, Epstein EJ, McKendrick CS, Galloway RW, Walker E. Systemic embolism in mitral valve disease. Br Heart f 1970; 32: 26-34.

${ }^{4}$ Casella L, Abelmann WH, Ellis LB. Patients with mitral stenosis and systemic emboli; hemodynamic and clinical observations. Arch Intern Med 1964; 114: 773-81.

${ }^{5}$ Langdell RD, Wagner RH, Brinkhous KM. Effect of antihemophilic factor on one-stage clotting tests. $L a b$ Clin Med 1953; 41 : 637-47.

${ }^{6}$ Ratnoff OD, Menzie C. A new method for the determination of fibrinogen in small samples of plasma. f Lab Clin Med 1951; 37: 316-20.

'Remmert LF, Cohen PP. Partial purification and properties of a proteolytic enzyme of human serum. f Biol Chem 1949; 181 : 431-48.

7aAlkjaersig N, Fletcher AP, Sherry S. The mechanism of clot dissolution by plasmin. $\mathcal{F}$ Clin Invest 1959; 38: 1086-95.

${ }^{8} \mathrm{McNicol}$ GP, Gale SB, Douglas AS. In-vitro and in-vivo studies of a preparation of urokinase. $\mathrm{Br} \mathrm{Med} \mathcal{F}$ 1963; 1: 909-15.

${ }^{9}$ Nilsson IM, Olow B. Fibrinolysis induced by streptokinase in man. Acta Chir Scand 1962; 123: 247-66.

${ }^{10}$ Merskey C, Kleiner GJ, Johnson AJ. Quantitative estimation of split products of fibrinogen in human serum, relation to diagnosis and treatment. Blood 1966; 28: 1-18.

${ }^{11}$ Breckenridge RT, Ratnoff OD. Studies on the nature of the circulating anticoagulant directed against antihemophilic factor: with notes on an assay for antihemophilic factor. Blood 1962; 20: 137-49.

${ }^{12}$ Bowie EJW, Owen CA Jr, Thompson JH Jr, Didi- sheim P. Platelet adhesiveness in von Willebrand's disease. Am f Clin Pathol 1969; 52: 69-77.

${ }^{13}$ Born GVR, Cross MJ. The aggregation of blood platelets. F Physiol (Lond) 1963; 168: 178-95.

${ }^{14}$ Spaet TH, Cintron J. Studies on platelet factor-3 availability. $\mathrm{Br} \mathcal{F}$ Haematol 1965; 11: 269-75.

${ }^{15} \mathrm{Holmsen} \mathrm{H,} \mathrm{Day} \mathrm{HJ,} \mathrm{Storm} \mathrm{E.} \mathrm{Adenine} \mathrm{nucleotide}$ metabolism of blood platelets. VI. Subcellular localization of nucleotide pools with different functions in the platelet release reaction. Biochim Biophys Acta 1969; 186: 254-66.

${ }^{16}$ Prentice CRM, Hassanein AA, McNicol GP, Douglas AS. Studies on blood coagulation, fibrinolysis and platelet function following exercise in normal and splenectomized people. Br $\mathcal{F}$ Haematol 1972; 23: 54152.

${ }^{17}$ Weily HS, Steele PP, Davies H, Pappas G, Genton E. Thromboembolism in rheumatic heart disease: correlation with platelet function studies (abstract). Circulation 1972; 45 and 46: Suppl. II 107.

${ }^{18}$ Aster RH. Pooling of platelets in the spleen: the role of the pathogenesis of 'hypersplenic' thrombocytopenia. f Clin Invest 1966; 45: 645-57.

${ }^{19}$ Kallinkos-Maniatis A. Megakaryocytes and platelets in central venous and arterial blood. Acta Haematol (Basel) 1969; 42: 330-5.

${ }^{20}$ Gryglewski RJ, Korbut R, Ocetkiewicz A. Generation of prostacyclin by lungs in vivo and its release into the arterial circulation. Nature 1978; 273: 765-6.

${ }^{21}$ Moncada S, Korbut R, Bunting S, Vane JR. Prostacyclin is a circulating hormone. Nature 1978; 273: 767-8.

${ }^{22}$ Piper $\mathrm{P}$, Vane J. The release of prostaglandins from lung and other tissues. Ann NY Acad Sci 1971; 180: 363-85.

${ }^{23}$ Rizza CR (1961). Effect of exercise on the level of antihaemophilic globulin in human blood. $\mathcal{f}$ Physiol (Lond) 1961 ; 156: 128-35.

${ }^{24}$ Taylor SH. The circulatory consequences of heart failure and shock. In: Hamer J, ed. Recent advances in cardiology, 6th ed. Edinburgh, London: Churchill, Livingstone, 1973: 211-34.

${ }^{25}$ Szekely $\mathrm{P}$. Systemic embolism and anticoagulant prophylaxis in rheumatic heart disease. $\mathrm{Br}$ Med $\mathcal{f}$ 1964; 1 : 1209-12.

${ }^{26}$ Sullivan JM, Harkden DE, Gorlin R. Pharmacologic control of thromboembolic complications of cardiac valve replacement. A preliminary report. New Engl f Med 1968; 279: 576-80.

Requests for reprints to Professor G P McNicol, University Department of Medicine, The General Infirmary, Leeds LS1 3EX. 